\title{
NON-RELATIVISTIC TIME, EXISTENCE AND ADAPTATION
}

\author{
R.G. BOOTHROYD \\ Retired Chartered Engineer, Queensland, Australia.
}

\begin{abstract}
By using non-dimensional Navier-Stokes equations in systems where partial modelling complications can be avoided, it is shown that geometrically similar systems can exhibit identical behaviour but according to different time scales where the latter are also within our own control. These models indicate that the physical nature of time is not related to a fundamental constant, but the passage of time can have a different but still constant value in a particular system of our own choosing. This observation also adds to our knowledge relating to the dichotomy of interpreting time either as a flowing parameter or, alternatively, just accepting it as a part of space-time. The observation adds evidence in favour of both viewpoints. The conclusions also seem relevant to biological species, chemical processes in general and other branches of physics. Using earlier work of Prigogine and others, it also appears that instantaneous time has important properties dividing past and future time into two segments which differ fundamentally in physical characteristics. This seems to explain the 'raison d'tre' of the second law of thermodynamics and the necessarily asymmetrical nature of time in our own world where mathematical symmetry of physical laws is the norm. This entropic time barrier also seems to explain the physically intangible nature of past events and their possible natural importance as former lower entropy forms of existence. It is concluded that these physical features of time are necessary for the existence of any form of life and Darwinian evolution, in particular. The conclusions may also help to throw further light on other suggested theories and observations in physics and the role of quantum decoherence in life and its adaptive ability.

Keywords: causality, cosmological time, entropy, evolution, fluid dynamic modelling.
\end{abstract}

\section{INTRODUCTION}

Recent advances in fundamental physics demonstrate that we live in a world of bewildering complexity. Whereas some topics illustrating this point, such as particle physics [1], are well covered, this is not the case in some other areas. Prominent among these is gravitation, a force field which cannot be shielded like electromagnetic fields, yet seems to emanate uniformly from all forms of matter as one of the smallest, but most important, of forces known to us. Our understanding of gravity has hardly advanced since Einstein's General Theory of Relativity.

The other great outstanding mystery in physics today is the nature of time itself, a subject which has intrigued us since the time of Aristotle [2]. Once again, our understanding of the nature of time is minimal despite the huge literature [3-7] devoted to it. This paper reports a relevant observation, which may lead to a better understanding of a subject that has puzzled us for centuries.

\section{FLUID DYNAMIC MODELLING}

This well-established technique is covered in most text-books on fluid dynamics [8,9], which include the use of Buckingham's ' $\pi$ ' theorem [10]. Many investigations of problems in science and technology require a partial modelling approach $[11,12]$. However partial modelling presents obfuscation in clarifying the conclusions in this paper. This is because when too many dependent variables are involved, these often entail their own separate and yet constant interacting time scales which would confuse our interpretation. To explain clearly how fluid dynamic models can help to elucidate the nature of time, it is best to revert to the original study based on the experiments of Reynolds [13]. 
3 SMOOTH DUCT FLOW AND THE DIMENSIONLESS NAVIER-STOKES EQUATIONS Incompressible fluid flow of a Newtonian liquid is expressed by the Navier-Stokes equations, shown here in vector form. The equations are non-linear.

$$
\rho\left(\frac{\partial \mathbf{v}}{\partial \mathrm{t}}+\mathbf{v} \cdot \nabla \mathbf{v}\right)=-\nabla \mathrm{p}+\nabla \cdot \mathbf{T}+\mathrm{f}
$$

where $\mathbf{T}$ is the stress tensor of the effect from viscosity.

Here we are mainly interested in the direction of flow down a pipe in the 'i' direction:

$$
\rho\left(\frac{\partial u_{i}}{\partial t}+u_{j} \frac{\partial u_{i}}{\partial x_{i}}\right)=-\frac{\partial p}{\partial x_{i}}+\mu \frac{\partial^{2} u_{i}}{\partial x_{j} \partial x_{j}}+f_{i}
$$

In dimensionless form, eqn (2) can be expressed with greater clarity [14]. The ' $\mathrm{f}$ ' forces are zero because there is no free surface in the flow.

$$
\frac{\partial u_{i}^{*}}{\partial t^{*}}+u_{j}^{*} \frac{\partial u_{i}^{*}}{\partial x_{j}^{*}}=-\frac{\partial p^{*}}{\partial x_{i}^{*}}+\frac{v}{U D} \frac{\partial^{2} u_{i}^{*}}{\partial x_{j}^{*} \partial x_{j}^{*}}
$$

In eqn (3), $u_{i}^{*}=u_{i} / U ; u_{j}^{*}=u_{j} / U ; t^{*}=t U / D ; x_{i}{ }^{*}=x_{i} / D ; x_{j}{ }^{*}=x_{j} / D ; p^{*}=p / \rho U^{2} ; v=\mu / \rho ; U D / v=N_{R e}$

It should be noted (Appendix I) that the term $\partial \mathrm{p}^{*} / \partial \mathrm{x}_{\mathrm{i}}{ }^{*}$ is a function of $\mathrm{N}_{\mathrm{Re}}$ only. It should also be noted that because the same eqn (3) is used for all models and the equation has the same boundary conditions in non-dimensional form, then the solutions to eqn (3) are identical for any particular value of $\mathrm{N}_{\mathrm{Re}}$. This applies to the solution in closed form (laminar flow) and also in turbulent flow where $\mathrm{u}_{\mathrm{i}}, \mathrm{u}_{\mathrm{j}}$ and $\mathrm{u}_{\mathrm{k}}$ are all statistically varying functions of time as well as space. In turbulent flow, solutions to eqns (2) and (3) have to be obtained numerically.

Use of eqn (3) does not depend on the cross-sectional shape of the pipe, although our examination will be restricted to flow well down a circular pipe where the boundary layers [15], whether laminar or turbulent, have merged and become fully developed. In this way, eqn (3) does not need to contain an extra dimensionless parameter in terms of $\mathrm{D} / \mathrm{L}_{\text {total }}$, the presence of which, again, would confuse the analysis.

It is seen that in terms of the dimensionless parameters used in eqn (3), the flow detail in dimensionless form depends only on the Reynolds number. Nevertheless, this claim has a caveat.

In the range $0 \leq \mathrm{N}_{\mathrm{Re}} \leq 2000$ where the flow is laminar and where the flow is turbulent $\left(2800 \leq \mathrm{N}_{\mathrm{Re}}\right)$, the conclusions arising from eqn (3) are valid. However, 2800 may become a higher value if steps are taken to delay the onset of turbulent instability. Where $\mathrm{N}_{\mathrm{Re}}$ lies in the transition range [15, p. 526, Fig. 20.1] where the flow changes over from laminar to turbulent, extra parameters causing instability, enter eqn (3). Typically these are pipe imperfections like roughness or small vortices (fluid circulation) which enter the pipe. Today we would describe this instability as a form of 'butterfly effect', interpretable by 'chaos' theory, but one which is in a very severely constrained system. The transition range of $\mathrm{N}_{\mathrm{Re}}$ brings in extra parameters such as pipe roughness effects into eqns (2) and (3) [8, p. 286, Fig. 5.32].

Now consider the flow of the same fluid in different-sized ducts but with the same Reynolds number. Because the kinematic viscosity $(\mu / \rho)$ is the same, then UD is the same in pipes with the same value of $\mathrm{N}_{\mathrm{Re}}$. If we consider two pipes denoted by suffices $\mathrm{A}$ and $\mathrm{B}$, then:

$$
\mathrm{U}_{\mathrm{A}} \mathrm{D}_{\mathrm{A}}=\mathrm{U}_{\mathrm{B}} \mathrm{D}_{\mathrm{B}}
$$


Also, because eqn (3) is the same for pipes A and B and because the pipe flows have the same boundary conditions in dimensionless form, then the solutions to eqn (3) must be identical. This conclusion applies if the solutions are in closed form (laminar flow) or are statistical (turbulent flow where the flow is expressed in statistical parameters). It so happens that these dimensionless statistical values do not vary much when $\mathrm{N}_{\mathrm{Re}} \geq 5 \times 10^{4}[15, \mathrm{p}$. 563].

The important conclusion is that any one of a series of such identical models can be considered to operate in real time. It then follows that all the other models behave identically but function in different time scales, either faster or slower. For the simple case of a Newtonian fluid flowing in a circular duct, it follows that these time scales are related by a very simple expression:

$$
\mathrm{T}_{\mathrm{A}} / \mathrm{T}_{\mathrm{B}}=\left(\mathrm{D}_{\mathrm{A}} / \mathrm{D}_{\mathrm{B}}\right)^{2}
$$

This expression applies to both laminar and turbulent flow.

Equation (5) and its derivation are significant. The derivation suggests that time is a flowing phenomenon, a conclusion which most of us would regard as mere common sense. However, it is quite common in modern physics for common sense and reality to fail to concur. Nevertheless, the observation that the passage of time can be varied by these models does not support the popular theory $[16,17]$ that time is a mere ingrained figment of the human imagination. Yet, the terms in eqn (5) are those which would be expected as they only relate to space-time. Thus, it seems that time can be interpreted and used both as a flowing phenomenon and also as a part of space-time. In physics, such dual conclusions are sometimes forced upon us. Wave-particle duality is probably the best known example which springs to minds.

\subsection{Experimental confirmation}

The theoretical approach described in Section 3 can be readily subjected to experimental confirmation. This is reasonably straightforward because this would be essentially just a much larger repeat of Osborne Reynold's original study, but using modern instrumentation. To be able to demonstrate, and particularly over a range of artificially induced time scales, that time is a constant, forwardmoving variable, is an important contribution to science and technology despite the fact that this is a supposition we have been using for many years. The various forms of turbulent correlation coefficients, Reynolds stresses, etc. would be expected to demonstrate similarity in all the models examined of different sizes [18, pp. 39-67].

Flow of a fluid in a pipe is a convenient way of transforming time to the spatial dimension. The past, the present and the future are all different positions of distance down the pipe, expressible approximately in terms of $\mathrm{U}$. The arrow of time is in the direction of fluid flow. For the limited conditions specified above, the flow in a pipe effectively freezes the past, present and future for observation into the same instant of time. For turbulent flow, where the increments in time are related statistically, this is an interesting facility in its own right.

Instrumentation techniques for studying turbulent flow have improved enormously in recent years and a laser-activated tracer technique [19] would be a vast improvement on early methods [20] used to avoid disturbing the flow from invasive instrumentation. Significant improvements in data analysis are also available in more recent work [21].

\subsection{Time scales in other dimensionless numbers}

Although it has been necessary to simplify the dimensional analysis in this paper to include $\mathrm{N}_{\mathrm{Re}}$ as the only, and yet most relevant variable, usually most engineering problems use partial modelling 
where the other dimensionless parameters are also often (but not always) found to be different time scales associated with other physical details. Sometimes these significant dimensionless parameters are too numerous [11] for the dimensional analysis to be useful. At other times, the time scales are too difficult to define mathematically. One such example is an elastic tube (in this case, a human artery) which is tethered elastically to other human tissue and also subjected to pulsatile flow [12]. The Womersley number used only goes part of the way in dealing with this problem.

Time scales have been found useful in other areas of biology. An interesting example [22] is the claim that a time scale based on 'mean generation time of species' instead of 'years of life' reduces 'noise' in the statistical analysis of data related to endangered species.

Also, it should be noted that ref. [12] used the normal technique of evaluating the order of magnitude of different parameters in order to assess their likely significance. The present study presents an alternative way of evaluating the likely significance of parameters in dimensional analysis. This is to examine their time scales in the analysis.

As an example, consider chemical reprocessing of spent nuclear reactor fuel elements. The processed mixture contains a large range of radioactive isotopes which decay at widely varying rates depending on the quantum effects. It is likely to be instructive to compare their half-lives with the time scales of the chemical reactions involved. It may be possible to ignore isotopes with short half-lives and regard isotopes with long half-lives as a constant factor in design analysis. In this way, we may be able to simplify a very complicated engineering design realistically.

\section{THE QUALITATIVE NATURE OF TIME}

The above fluid dynamic approach expresses time scales as ratios in terms of other parameters, but this tells us nothing else about the present time in which we live. At this stage, we can only conclude that time, be it real, or even artificially engendered in the human mind, always appears to move at a constant rate, but it does not appear to be related to a fundamental constant like G or c.

Clearly we need to extend our investigation. Prigogine and Stengers [23] have drawn attention to the possibility of a relationship of time with the velocity of light given by Maxwell's relationship:

$$
\mathrm{c}=\left(\varepsilon_{0} / \mu_{0}\right)^{-1 / 2}
$$

but this seems to have led nowhere.

In advancing our investigation, it is considered that our prime need is for a plausible physical model on which a detailed mathematical model and analysis can be based. Previous studies [23-26] indicate that we are well-equipped with regard to mathematical techniques. However, as shown below, this is not the case because we need to use non-equilibrium thermodynamic calculation techniques and this subject is still in its infancy [27]. The likely success of mathematical modelling is critically dependent on the choice of model we seek to analyse. Therefore, the rest of this paper will concern itself with this necessary preliminary stage of further research.

The role of the explorative experimental physicist is a vital precursor to any full analysis by mathematical physicists. This is needed in the present study as mentioned in section (7.1). Experimental facts need to be verified by laboratory physicists first and tested rigorously with an investigation of their likely range of application. Strange anomalies need to be reported, including suspicions concerning possible singularities and complex numbers which may have far-reaching effects in mathematical analysis.

The simplified preliminary discourse in Appendix II may help the readers in following the arguments to be suggested.

To our earthly experience, time can be divided into three parts: the past, the present and the future. From the point of view of the physicist, they seem to be three very different regimes. 


\subsection{The past}

The main characteristic of the past is its permanence. If we were to keep comprehensive historical records, we would be able to analyse all past events in terms of classical Newtonian mechanics. Quantum effects are apparent, but they are frozen in time to make causality certain and examinable. Perhaps the most important and valuable quality of the past is that it appears to be dead and in a permanent state. It is fully deterministic. Its entropic state as a whole system is always less than (or at the most equal to) the entropy of the immediate present. Its entropic state is always less than that in the future.

The past is of enormous value to a cognizant species such as Homo sapiens in that it affords us the facility of experience to plan for events in an uncertain and indeterminate future. For other biological species, the past is potentially a detailed record of the mechanism of their automatic Darwinian adaptation to their own uncertain future.

\subsection{The future}

The future is so very different from the past. It is hardly ever deterministic, but is subject to widespread forms of statistical uncertainty. Disease, fickle climate and conflict are typical examples. To a small extent, uncertainty is limited when probability can be neglected for practical purposes. The behaviour of the solar system is one such example, although it also changes slowly. Most of our future experiences are statistically based. The future is also the true realm of quantum physics, natural biological control systems and uncertainty. We can make plans for our own future, but only with detailed analysis of the random nature of natural processes. The future is always associated with increase of the entropic state of the whole system.

\subsection{The present}

The present, or instantaneous time, divides the past from the future. Because the past and the future are so very different, consideration of the present instant suggests to us to that the present time, although very short in duration, must have some very significant and unusual qualities which may not be obviously apparent to us. We need to ask ourselves what are the physical processes at work in present time? Using our own concept of reality, the present appears to move with our own existence continuously and at a constant rate. It appears to separate the past from the present and sweep all that still exists into an uncertain future. Although it may seem intangible to us, instantaneous time suggests to us that it is likely to be a barrier between the past and the very different future. Physically it is certainly a very pronounced discontinuity. Viewed very simplistically, the present seems to be a moving temporal barrier between the past and the future and one where all processes, be they physical, chemical or biological, change dramatically. Perhaps this barrier may become expressible in units of Planck time?

$$
\mathrm{t}_{\mathrm{p}}=\left(\mathrm{hG} / \mathrm{c}^{5}\right)^{1 / 2}=5.39 \ldots \times 10^{-44} \mathrm{~s}
$$

The present is where the frozen immobility of the dead past suddenly presents itself as still alive and becomes subject to statistical mechanics and the second law of thermodynamics involving increasing entropy and uncertainty. It is this natural moving coordinate system which needs close theoretical scrutiny.

\section{IMPORTANCE OF PRIGOGINE'S WORK}

The above reasoning that instantaneous time is likely to be an entropic barrier separating past time from future time is based largely on the selected opinions of several, but not all, scientists. Prigogine 
and Stengers [23] have used very different arguments to come to the same conclusion [23, pp. 295-296] that an entropic barrier is an important aspect of instantaneous time in its presumed function to separate the past from the future. Prigogine's research has received considerable independent support [28]. This agreement is encouraging.

Any attempt at theoretical examination of the future does not seem to be promising because, by its nature, the future cannot yield firm data. On the other hand, comparing the past with the present may help in our understanding. In examining the past, it seems that the 'sliced loaf of bread model' [29, pp. 57-61, pp. 130-139], which is a development of the 'block' model, seems to be particularly useful in investigating an entropic time barrier model.

One likely consequence of this model is that the entropic transfer barrier from the present to the past seems to be formidably strong compared with the similar entropic barrier if moving from the past to the present. It can be likened to a non-return valve except that it is one controlled by entropy (and/or some other factor such as possibly a quantum decoherence effect - see section 7.1). The forward barrier exists, but transfer across this barrier is from a low entropy state to a higher one, which is easier thermodynamically This barrier is especially weak for an inanimate object which, in an isothermal transfer, requires almost no entropy change at all in making a transfer, but the barrier is much stronger for a living object which needs to excrete entropy to the environment during any suggested possible transfer. Thus, the possibility of transfer from the present to the past appears to be by far the most difficult effect to achieve. This may be because each increment (obliquely sliced segment of the sliced loaf of space-time [29, p. 135]) of past time isolates the passing increment of time from the adjacent increment which succeeds it in forward-moving time. This barrier can, perhaps, be considered to be a multi-layered one, thus making it likely to be a very strong one indeed.

In conclusion, it seems likely that any living creature (and this includes even a well-designed automated machine - which also would have entropy dispersal needs) cannot pass from the present to the past. It seems that this may explain away the paradox of a person returning to the past to kill his/her own grandfather. It seems that even a very cleverly designed machine could only have a very low penetration into the past. It may be reassuring for us that our ancestors appear to be safe in their graves. Nevertheless, it is necessary to carry out calculations to clarify this supposition.

Perhaps the strongest evidence for the strength of this backward moving part of the entropic time barrier is the oft-quoted question, 'Where are the tourists from the future?'

When we consider the possibility of travelling from the present to the future, a similar entropic barrier exists, although it seems to be less of a hurdle as it is from a lower entropy state to a higher one. However, it has been pointed out that such transfers are not just a matter of net entropy transfers, but the rate at which entropy can be transferred becomes important [30]. This is why we cannot avoid using the latest developments [27] in non-equilibrium thermodynamics when making calculations. Thus, the possibility of such a transfer may be limited by the size of the time barrier, if it can be shown to exist.

It seems that although time travel to the future may be difficult, it cannot be ruled out as a possibility. However, it has been pointed out [3] that the future may not contain any events for us to visit. The main problem, however, seems to be that time travel to the future seems likely to be a one-way trip because of the seemingly much stronger barrier preventing return.

Physicists remain perturbed [29, pp. 128-142, 31] by the fact that the behaviour of time is almost unique among our physical laws in not being symmetrical about itself (time reversal invariant). It has even been suggested that if time can be reversed, then entropy should increase as time moves backwards [29, p. 161, Fig. 6.2b]. This has been questioned [32].

It seems possible that the natural symmetry of time may have changed at some stage in early creation towards a forward-moving arrow of time. However, opinions vary in such a difficult 
speculation [33]. Our world has endured many strange events before it became fit for intelligent lifeforms to develop. Sometimes we have plausible explanations such as the necessary demise of the dinosaurs to make way for the development of mammalian life. Usually reasons for past events remain shrouded in mystery.

\section{PRESENT CONTROVERSY RELATING TO OUR INTERPRETATION OF THE NATURE OF TIME}

The observations in section (3) suggest that time can be interpreted either as space-time as elucidated by Einstein's General Theory of Relativity (this is the Parmenidean School) or alternatively, time can be interpreted as a flowing phenomenon. These are the Heracliteans [3]. Normally different people subscribe to one or the other of these viewpoints and only a minority seem to subscribe to both.

Design engineers are always 'Heracliteans' [3], although few professional engineers would have ever heard of the term. Design engineers use a whole range of equations expressed in terms of time as a constantly flowing continuous parameter (the 'time flux' point of view). They integrate and differentiate these expressions with success in different branches of technology. Often these design equations are of an empirical nature with limited scientific basis. It is sufficient for these Heracliteans that their designs can be brought to a satisfactory and logical conclusion.

On the other hand, the majority of physicists seem to be 'Parmenideans' [3]. Only a few [3,34] declare themselves openly to have Heraclitean sympathies. Parmenideans assert that time does not flow at all, and some even suggest that time does not even exist and is a mere figment of human imagination [16,17]. Many Parmenideans are eminent scientists [29, 35] and include Einstein himself. They support the 'stasis' view of the 'block universe'. The pedigree of the Parmenideans is beyond reproach because their viewpoint is to examine all existence from outside of time and space. This is the presumed divine creator viewpoint, expressed so lucidly by C.S. Lewis [36]. This metaphysical viewpoint has a history many centuries old including contributions from theology scholars and other distinguished philosophers.

It seems reasonable to conclude that a deeper understanding of the nature of time is needed.

\section{SUGGESTED OUTLINE OF FURTHER INVESTIGATION}

Statistical mechanics and non-equilibrium thermodynamics [27], in particular, can be used to calculate the desirable width of an entropic barrier in terms of Planck time. This would be on a probability basis, i.e. the chance that instantaneous time can form an effective barrier to a prescribed transfer process. Calculations would involve different materials and different particles. It is not sufficient to merely examine the transfer of particles. It is also necessary to evaluate their ability to transfer information. Information $[37,38]$ has been described as negative entropy. Fundamental particles can pop into and out of existence all the time, but their ability to carry information is limited. This suggests that the required width of the entropic barrier could be best evaluated for a small virus with an estimation of the probability of its survival in an entropic transfer. This may be of basic interest because a virus is a basic form of information. Can a virus cross an entropic time barrier from the present to the past and vice versa? This can be compared with the smaller width barrier needed to block transfer likely to be related to more sophisticated lifeforms. These calculated widths would be of much interest compared with the statistical consequences of a different entropic width which might actually exist. In other words, is a natural entropic width compatible with the needs of our earthly existence? Would this throw light on the time scale of the natural flow of time which we experience? What is the actual probability of intact information transfer for different conditions? Would this information be related to other suspicions 
suggesting that the exact requirements for a 'platform for life' are being met [39-41]? Other suspicions that Prigogine's entropic barrier may be more than a discontinuity and may exhibit singularity properties and complex numbers arising from quantum considerations are also worth bearing in mind.

7.1 The need for prior experimental investigation before investigating Prigogine's entropic barrier model

The present writer is unaware of any serious laboratory investigation into the physical properties of instantaneous time. This may be for a very good reason that the experimental difficulties are too great. Research into the allied field of quantum tunneling [42] suggests that this is the case. Quantum tunnelling is very fast, apparently exceeding the speed of light (although careful examination has shown that this does not violate relativity and causality considerations).

Prigogine's approach may be the best candidate for finding explanations, but there may be other explanations of the strange physical nature of instantaneous (present) time. These may be of a quantum mechanical nature based on Schrodinger's wave equation and decoherence effects [43]. Before embarking on an expensive investigation into suspected entropic time barriers, it seems to be wise to carry out some laboratory studies first. We have a range of equipments and techniques for measurements over short intervals of time, etc. to help us to investigate the minimum time interval needed for an entropic time barrier to explain the transition of present time to past time. If there is no experimental evidence at all for finite time intervals in the nature of instantaneous time, then it would be preferable to delay an expensive numerical investigation into entropic time barriers in favour of finding an alternative quantum explanation. Quantum effects are expected to act almost instantaneously and much faster than an entropic effect. It is difficult to restrain quantum effects from collapse and an event lasting $10^{-4} \mathrm{~s}$ is difficult to achieve [44]. Other fundamental studies in solid state physics may also be useful in this investigation.

At this early stage, it is also important to consider that decoherence-controlled and entropycontrolled transfer processes may even act together jointly in giving us the mechanism of a suspected barrier in time. When we know so little, it seems wise to keep our options open.

Many quantum processes are time-reversible. For example, a container of inert gas like neon can, theoretically, be kept for all eternity at is maximum steady entropy but still experiencing statistically varying conditions, as the molecules collide elastically with each other. Yet, quantum physics also has some very irreversible phenomena such as radioactivity and wave collapse/decoherence. It is surmised that this complexity may be an important ingredient in our 'platform for life'.

Furthering our understanding of the physical nature of time would be an important contribution towards our understanding the 'platform for life' on which we all depend. Our very existence seems to be the result of a long sequence of statistically improbable freak events [39-41]. If we are eventually forced into accepting teleological explanations for our existence, we should at least have tried hard first to elucidate and understand the scientific basis for our 'platform for life'.

\subsection{The need for plausibility in developing a theoretical model}

Much of the research in modern physics involves phenomena (and even presumptions) which we cannot see. For example, only recently has it become possible to see the statistical variation of the orbiting electron in a hydrogen atom [45]. In another example, Schrodinger's wave function appears to be no more than a mathematical abstraction, and yet its application generates realistic results which can be confirmed experimentally. 
What is needed for elucidating Prigogine's entropic barrier is a plausible and well-founded theoretical basis. It is likely that extensive numerical methods will be needed to examine such a theory. Altering parameters in computer calculations can throw considerable light on the accuracy of any theory and may even improve our ability to develop the theory. But this is only possible if the basic theory is plausible in the first place and includes all relevant parameters. Repeated calculations will sometimes close such a study to formulate reliable equations. These methods are lengthy and expensive as mentioned in an earlier recent paper [46, pp. 451-453] regarding optimisation of a new technology, which we will probably be forced to develop as a consequence of adverse influence on world climate.

\section{DISCUSSION}

Looking objectively at our own existence, we find that we are compelled to live lives which are very severely constrained. Our best form of communication (by electromagnetic waves) is tediously slow at $3 \times 10^{8} \mathrm{~m} \mathrm{sec}^{-1}$. It limits us to viewing much of the history of the cosmos which is only long-past. At its present rate of universe expansion, it seems that we are destined to even lose this part of history. We might well look with envy at quantum-entangled particles and their apparently instantaneous communication. In another example of our limitations, almost all of the entire chemistry of our bodies is controlled only by the selective power of outer electrons in the atoms we have inside us. Yet, perhaps, the greatest constraint in our lives is the second law of thermodynamics which chains us to entropy and the forward arrow of time.

Yet, recognising our own humble status as mere integrated examples of organic chemistry, perhaps we should be grateful for our stable and secure 'platform for life'. Our 'platform for life' has its set of scientific rules resulting in causality, so that we can be logical in explaining our experience and managing our affairs. Most of these rules are workable rules in classical Newtonian mechanics. For example, these rules are sufficient for us to engineer an automated exploration of the planets in our solar system which is an impressive achievement. Yet, it is mainly the field of quantum physics which shows us that rules often have exceptions and often these exceptions seem to be necessary.

\section{CONCLUSIONS}

It is concluded that when comparing some similar systems, time can be shown to move faster or slower than the time scale in which we live. Although, like c, time is a constant in our lives, it seems that it is not related to a fundamental constant like $\mathrm{G}$, the gravitational constant.

Studying fully developed turbulent flow transforms time events of past, present and future into sequentially identifiable positions down a long tube. At any particular instant, the past, present and future exist together and are related with each other statistically, but each observation at any instant can be engineered to a time scale to suit us.

Prigogine's earlier research seems to be a promising line of investigation to take in explaining the true nature of time by evaluating the influence of entropy as an explanation of the strange time asymmetrical phenomena which we observe. It is also reasoned that time, at the present instant, presents a strong discontinuity between the past and the future because of the very different physical nature of time-past and time-future. By way of explanation, there are also suspicions that a quantum decohesion effect, which we do not yet understand, may also be involved in the discontinuity effect in instantaneous time.

Clearly, this topic and its consequences deserve a more detailed examination than can be given here. It is proposed to review the subject again in 5 years [47]. Five years may seem a long time in a fast-moving scientific era, but it is short in comparison with several hundred years of mystery attached to the subject of time. 
It seems that time can be examined realistically in two ways. Time is examinable as a part of space-time, which is necessary if relativistic effects are felt. Alternatively, in our everyday world, the concept of time as a moving parameter helps us to understand and manipulate our world. The conclusions resulting from section (3) support this. Both the Parmenidean and Heraclistic viewpoints are useful.

As a final conclusion, the forward-flowing nature of time is clearly a most important factor in understanding the mechanisms at work in our 'platform for life'. This platform is so intricate, complicated, elegant and sophisticated that it is hard to avoid the suspicion that it is also contrived.

\section{APPENDIX I: $\partial \mathrm{p} * / \partial \mathrm{x}_{\mathrm{i}} *$ IS A FUNCTION OF $\mathrm{N}_{\mathrm{Re}}$ ONLY}

Consider a length $x_{i}$ of circular pipe with diameter $D$. This incurs a pressure drop $p\left(x_{i}\right)-p(0)$, where $p(0)$ is the pressure where $x_{i}=0$. In this region, the boundary layers are fully developed, and therefore, $\mathrm{p}\left(\mathrm{x}_{\mathrm{i}}\right)$ varies linearly with $\mathrm{x}_{\mathrm{i}}$. Also, $\tau$, the wall shear stress on the fluid, is constant.

Balancing the forces on a moving cylindrical element of the fluid (which is not accelerating), we have

$$
\pi \mathrm{D}^{2}\left(\mathrm{p}\left(\mathrm{x}_{\mathrm{i}}\right)-\mathrm{p}(0)\right) / 4=\pi \mathrm{Dx}_{\mathrm{i}} \tau
$$

where $\partial \mathrm{p}\left(\mathrm{x}_{\mathrm{i}}\right) / \partial \mathrm{x}_{\mathrm{i}}=4 \tau / \mathrm{D}$.

The wall shear stress $(\tau)$ for a smooth pipe is given by

$$
\tau / \rho \mathrm{U}^{2}=\Phi\left(\mathrm{N}_{\mathrm{Re}}\right)=\mathrm{f},
$$

the friction factor $[8$, p. 288].

Thus, $\partial \mathrm{p} / \partial \mathrm{x}_{\mathrm{i}}=4 \rho \mathrm{U}^{2} \Phi\left(\mathrm{N}_{\mathrm{Re}}\right)$ and

$$
\partial \mathrm{p}^{*} / \partial \mathrm{x}_{\mathrm{i}}^{*}=4 \Phi\left(\mathrm{N}_{\mathrm{Re}}\right)
$$

i.e. a function of $\mathrm{N}_{\mathrm{Re}}$ only.

Note also that $\mathrm{U}$ is normally taken as the mean axial velocity in the pipe evaluated from integration of the radially varying velocity profile in the direction of the pipe axis.

For laminar flow, $\mathrm{u}_{\mathrm{i}}(\mathrm{r})$ is parabolic and for turbulent flow, the time mean average of $\mathrm{u}_{\mathrm{i}}(\mathrm{r})$ is taken from experimental data. Any other consistently used and reasonable definition of $\mathrm{U}$ is suitable.

\section{APPENDIX II: SIMPLIFIED OUTLINE OF A PROPOSED BASIS FOR PHYSICAL/ MATHEMATICAL ANALYSIS INTO INSTANTANEOUS TIME AND PAST TIME}

The natural coordinate system for any mathematical analysis of time seems to be a moving Lagrangian coordinate system which follows us exactly. Instantaneous time (now) also seems to be bounded by two barriers.

- Future time which is subject to all the vagaries of statistics (and almost all of our very existence is statistically based). Future time seems, therefore, to be the most unattractive as a direct basis for analysis.

- By contrast, the supposed barrier between instantaneous time and past time has hard and determinate facts against which we can test any theory. Therefore, this seems to be the logical path to take in research. 
- The approach is best illustrated by the following story about 'grandma and her chair' and how this is affected by the lower entropy state of the immediate past and the higher entropy state of the present time.

Grandma's chair has no difficulty in travelling intact from the past to the present. The chair is inanimate and large. The chair's entropy has hardly changed at all in passing from the past to the present. Quantum effects are immeasurably small when considering large inanimate objects. How does this compare with Grandma herself when she died? Certainly, Grandma's body had no difficulty moving from the past to the present. Nevertheless, its entropy increased considerably in moving from the past to the present. So, for a different reason, her body had no difficulty in moving from a lower entropy past to a higher entropy present. But what about the life of Grandma which was clearly debarred from entering the present? Throughout her life, Grandma kept her entropy more or less constant by increasing the entropy of her immediate environment. As she got older, this ability declined. She probably died when her immune system faltered and with this, her ability to control her entropy declined.

However, all this speculation is very complex, so let us consider a very small piece of matter which is easier to examine mathematically.

Many small pieces of matter are in a state of thermodynamic equilibrium where entropy is at its maximum possible value. Such an element would have no trouble moving from the past to the present (which is at a higher general entropy). In this case, no significant barrier seems to exist between the immediate past and the present.

It seems that for small objects not in a state of maximum possible entropy, the probability of moving from the past to the present or vice versa can be evaluated mathematically using statistical mechanics. Thus, if entropy conditions are sufficiently favourable, crossing the presumed time barrier may be possible.

It seems that the 'temporal width' of this barrier can be estimated (perhaps in units of Planck time). This temporal width is clearly a variable depending on the physical properties of the particle of matter being considered and its surroundings. It seems that life-forms, even very small viruses, sometimes cannot pass this barrier except with a very small probability.

At this stage we can suspect that, although the barrier between immediate past and present in invisible, it exists and it can be examined mathematically using statistical mechanics. The barrier seems to depend on the object considered and whether the object is inanimate or living. The supposed temporal width of the barrier is an important parameter because this affects the probability of passing between past and present and vice versa, and this probability seems likely to decrease as the past recedes in real time from the present.

\section{NOMENCLATURE}

$\mathrm{c}=$ velocity of light (eqn (6) where $\varepsilon_{0}$ and $\mu_{0}$ are the permittivity and permeability of free space, respectively)

$\mathrm{f}=$ friction factor $\tau / \rho \mathrm{U}^{2}$. N.B.: in eqns (2) and (3), f refers to a non-existent force

$\mathrm{L}_{\text {total }}=$ distance down the pipe where boundary layers have merged to give uniform flow

$\mathrm{N}_{\mathrm{Re}}=$ pipe Reynolds number UD/v

$\mathrm{p}=$ pressure in pipe

$\mathrm{t}, \mathrm{t}_{\mathrm{p}}=$ real and Planck time

$\mathrm{T}_{\mathrm{A}} / \mathrm{T}_{\mathrm{B}}=$ relative time scale ratio in pipes $\mathrm{A}$ and $\mathrm{B}$

u.v, = fluid velocities in pipe

$\mathrm{U}=$ average axial flow velocity 
$\mathrm{x}=$ axial position in pipe

$\mathrm{D}=$ circular pipe diameter $\left(2 \mathrm{r}_{\max }=\mathrm{D}\right)$

$\rho=$ density of fluid

$v=\mu / \rho$

$\mu=$ viscosity

$\tau=$ wall shear stress of pipe on fluid (negative in coordinate system used)

$\Phi=$ functional relationship

\section{REFERENCES}

[1] Martin, B.R., Particle Physics, Oneworld: Oxford, pp. 1-201, 2011.

[2] Davies, P.C.W., About time - Einstein's unfinished revolution, Penguin: London, p. 29, 1995.

[3] Weinert, F., The march of time-changing conceptions of time in the light of scientific discoveries, Springer: Dordrecht, pp. 1-281, 2013.

[4] Hawking, S. \& Penrose, R., The nature of space and time, Princeton University Press: Princeton, pp. 1-156, 2010. doi: http://dx.doi.org/10.1515/9781400834747

[5] Zeh, H.D., The physical basis of the direction of time (Edn 5), Springer: Berlin \& Dordrecht, pp. 1-240, 2007.

[6] Reichenbach, H., Direction of time, University of California Press, pp. 1-280, 1956.

[7] Gibbons, G.W., The emergent nature of time and the complex numbers in quantum cosmology, In Mersini-Houghton, L. \& Vaas, R.,(eds) The arrows of time: a debate in cosmology, pp. 109-145, Springer: Dordrecht, 2012. doi: http://dx.doi.org/10.1007/978-3-642-23259-6_6

[8] Streeter, V.L. Fluid mechanics, $5^{\text {th }}$ Edn, McGraw Hill: New York, pp. 201-231, p. 286, p. 288, 1971.

[9] Kay, J.M. \& Nedderman, R.M., An introduction to fluid mechanics and heat transfer, $3^{\text {rd }}$ Edn, Cambridge University Press, pp. 56-71, 1974.

[10] Buckingham, E., Model experiments and the form of empirical equations, Trans ASME, 37, pp. 263-296, 1915.

[11] Boothroyd, R.G., Similarity in gas-borne flowing particulate suspensions, Trans. ASME (Amer. Society of Mechanical Engineers (Journal of Engineering for Industry)), 91(2), series B, pp. 303-314, May, 1969.

[12] Hutton, P., Foster, K., Ashton, F. \& Boothroyd, R.G, An appraisal of a modelling technique for arterial flows, Engineering in Medicine, 4(3), pp. 3-12, 1976. doi: http://dx.doi.org/10.1243/ EMED_JOUR_1975_004_022_02

[13] Reynolds, O., An experimental investigation of the circumstances which determine whether the motion of water shall be direct or sinuous and the laws of resistance in parallel channels, Trans. Roy Soc. London, 174, pp. 935-982, 1883. doi: http://dx.doi.org/10.1098/rstl.1883.0029

[14] Kopera, M., Non dimensionalisation of the Navier-Stokes equations, Centre for Scientific Computing, University of Warwick www2.warwick.ac.uk/fac/sci/eng/study/.../ non-dimentionalization.pdf

[15] Schlichting, H., Boundary layer theory, McGraw Hill, pp. 1-747, 1968.

[16] Barbour, J., The end of time: the next revolution in our understanding of the universe, Weidenfeld \& Nicholson: London, pp. 1-371, 1999.

[17] Slezak, M., The time delusion, New Scientist, 219(\#2937), pp. 34-38, 2nd November. 2013. doi: http://dx.doi.org/10.1016/S0262-4079(13)62591-9

[18] Hinze, J.O., Turbulence an introduction to its mechanism and theory, McGraw Hill: New York, pp. 39-67, 1959. 
[19] Tropea, C., Yarin, A.L. \& Foss, J.F., (eds) Springer handbook of experimental fluid mechanics (vol. 1) Springer: Dordrecht, pp. 366-368, 2007. doi: http://dx.doi.org/10.1007/978-3-54030299-5

[20] Boothroyd, R.G., An anemometric isokinetic sampling probe for aerosols, J. Sci Instruments, 44, pp. 249-253, 1967. doi: http://dx.doi.org/10.1088/0950-7671/44/4/301

[21] Choi, J-I, Yeo, K, \& Lee, C., Lagrangian statistics in turbulent channel flow, Physics of Fluids, 16(3), pp. 779-794, 2004. doi: http://dx.doi.org/10.1063/1.1644576

[22] Frankham, R. \& Brook, B.W., The importance of time scale in conservation biology and ecology, Ann. Zool. Fennici 41, pp. 459-463, 2004.

[23] Prigogine, I. \& Stengers, I., Order out of chaos: man's dialogue with nature, Bantam: Toronto, pp. 257-296, 1984.

[24] Prigogine, I., From being to becoming: time and complexity in the physical sciences, Freeman: San Francisco, pp. 1-272, 1980.

[25] Prigogine, I., Introduction to thermodynamics of irreversible processes ( 2 nd Edn) Interscience: New York, pp. 1-119, 1961.

[26] Prigogine, I., Non equilibrium statistical mechanics. Interscience: New York, pp. 1-319, 1962.

[27] Attard, P., The second entropy: a general theory for non-equilibrium thermodynamics and statistical mechanics, doi 10.1039/b802697c, Ann. Rep. Prog. Chem. (C), 105, pp. 63-173, 2009.

[28] Coveney, P. \& Highfield, R., Arrow of time: a voyage through science to solve time's greatest mystery, Allen: London, pp. 161-378, 1990.

[29] Greene, B., The fabric of the Cosmos, Penguin: London, pp. 1-569, 2004.

[30] Gold, T., \& Bondi, H., (eds) The nature of time, (mtg May-June, 1963) Cornell University Press: New York, p. 229, 1967.

[31] Lederman, L.M. \& Hill, C.T., Symmetry and the beautiful universe, Prometheus: New York, pp. 1-363, 2004.

[32] Penrose, R., The road to reality: a complete guide to the laws of the Universe, Vintage books: London, p. 698, 2004.

[33] Harland, D.M., The big bang: a view from the $21^{\text {st }}$ century, Springer Praxis: Chichester, UK, p. 233, 2003.

[34] Smolin, L., Time reborn: from the crisis of physics to the future of the universe, Allen Lane: London, pp. 1-305, 2013.

[35] Davies, P.C.W.D., Stirring up trouble, in Halliwell. J.J., Perez-Mercader, J. \& Zurek, W.H., (eds) Physical origins of time asymmetry, Cambridge University Press, pp. 119-130, 1994.

[36] Lewis, C.S., Mere Christianity, Collins: London, pp. 145, 1944.

[37] Jarzynski, C., Equations and inequalities: irreversibility and the second law of thermodynamics at the nanoscale, In Duplentier, B., (ed) Progress in mathematical physics (vol. 63) (15 th Poincare' seminar, 2010 Springer: Basel, pp. 145-168, 2013.

[38] Fromhold, A.T., Jr, Quantum mechanics for applied physics and engineering, Academic Press: New York, pp. 237-278, 2012.

[39] Wilczek, F., The lightness of being: big questions, real answers, Penguin, London, pp. 1-270, 2008.

[40] Rees. M.R., Just six numbers, Weidenfeld \&Nicholson: London, pp. 1-194, 1999.

[41] Denton, M.J., Nature's destiny, Simon \& Schuster: New York, pp. 1-454, 1998.

[42] Prutchi, D., Do it yourself quantum physics: exploring the history, theory and applications of quantum physics through hands-on projects, J Wiley: Hoboken, pp. 178-180, 2012. doi: http:// dx.doi.org/10.1002/9781118170717 
[43] Duplantier, B., Raimond, J-M. \& Rivasseur, V., Quantum decoherence Poincare Seminar, (Progress in mathematical physics V 48), Springer: Birkhauser Verlag and Dordrecht, pp. 1-201, 2006.

[44] Al Khalili, J., Quantum: a guide for the perplexed, Orion: London, p. 249, 2014.

[45] Anon. Smile hydrogen atom you're on quantum camera, New Scientist, 218(\#2919), p. 13, June $1^{\text {st }}, 2013$. (Stodolna et al. Physical Review Letters, doi.org/mmz).

[46] Boothroyd, R.G. Proposed Australian transition to an anhydrous ammonia fuel transport economy to replace liquid petroleum fuel, WIT trans. Ecology and the environment, 186, pp. 443-456, 2014.

[47] Boothroyd, R.G., On the physical nature of instantaneous and historical time: a review, boothroyd.org, 2020. 